\title{
LEGIONELLOSIS: A WALK-THROUGH TO IDENTIFICATION OF THE SOURCE OF INFECTION
}

\author{
Dimosthenis Chochlakis ${ }^{1,2}$, Vassilios Sandalakis ${ }^{1,2}$, Maria Keramarou ${ }^{1,2}$, Yannis Tselentis², Anna Psaroulaki ${ }^{1,2}$ \\ ${ }^{1}$ Regional Laboratory of Public Health of Crete, Heraklion, Crete, Greece \\ 2Laboratory of Clinical Bacteriology, Parasitology, Zoonoses and Geographical Medicine, University of Crete, Heraklion, Crete, Greece
}

\section{SUMMARY}

Objectives: Although a number of human Legionnaires' disease in tourists are recorded annually in Europe, there are few cases where a direct link can be made between the infected person and the source of infection (hotel or other accommodation). We present a scheme followed in order to track down and identify the source of infection in a tourist suffering from L. pneumophila sg 5 infection, who was accommodated in seven different hotels during his holidays in the island of Crete, and we comment on various difficulties and draw-backs of the process.

Method: Water samples were collected from the seven hotels where the patient had resided and analyzed at the regional public health laboratory using cultivation and molecular tests.

Results: Of 103 water samples analyzed, $19(18.4 \%)$ were positive for Legionella non-pneumophila and $8(7.8 \%)$ were positive for $L$. pneumophila. A successful L. pneumophila sg 5 match was found between the clinical and environmental sample, which led us to the final identification of the liable hotel.

Conclusion: Timely notification of the case, within the the European Legionnaires' Disease Surveillance Network (ELDSNet) of the partners involved, is crucial during a course of travel associated with Legionella case investigation. Moreover, the urinary antigen test alone cannot provide sufficient information for the source identification. However, acquiring clinical as well as environmental isolates for serogroup and SBT identification is highly important for the successful matching.

Key words: Legionella pneumophila sg 5 , travel associated disease, surveillance, biotyping, urinary antigen test

Address for correspondence: D. Chochlakis, Regional Laboratory of Public Health of Crete, School of Medicine, University of Crete, Stavrakia, 70013, Heraklion, Crete, Greece. E-mail: surreydimos@hotmail.com

https://doi.org/10.21101/cejph.a4660

\section{INTRODUCTION}

The island of Crete is one of the most popular tourist destinations in Greece during summer, with thousands of people visiting during the season from April to October. Every year, sporadic cases of Legionnaire's disease occur during the touristic season.

Herein, we present a scheme followed in order to track down and identify the source of infection by L. pneumophila sg 5 in a tourist who was accommodated in seven different hotels during his holidays in the island of Crete. We also document various difficulties and draw-backs of the process.

\section{Case Notification}

In late July 2011, a French tourist visited the island of Crete. He spent 14 days in total there, staying at seven different hotels in all four prefectures of the island. Upon his return to France (8th of August), the patient was diagnosed positive for L. pneumophila infection by urine test at the hospital where he was admitted. A case report was issued to the European Legionnaires' Disease Surveillance Network (ELDSNet) on the same day by the French authorities. Further samples from the patient were collected within next days during his stay. Further clinical samples were cultivated and L. pneumophila sg 5 was isolated and identified.
On 1 September 2011, ELDSNet notified the Hellenic Centre of Disease Control and Prevention (HCDCP) in Athens, of a single confirmed case of Legionnaire's disease in a 65 year old male from France, who had stayed in seven different hotels in the island of Crete from 26 July to 8 August 2011. On 2 September, the Regional Laboratory of Public Health of Crete (RLPHoC) received notification of the case mentioned above through the Greek health authorities (Fig. 1).

\section{MATERIALS AND METHODS}

\section{Environmental Sampling}

Environmental investigations were initiated on 6 September by the local Greek authorities (Fig. 1). A total of 103 water samples were collected from all seven hotels in Crete, where the patient resided for at least one night. Six to 19 water samples were collected from each hotel depending on the architecture and system of water supply network and size of the hotel. Collection sites most commonly included: one water sample (tap water) at the inlet of each hotel water system, one right after the main water supply tank, one before and one after the water heater. Four water samples from room showers (direct cold and hot water samples, 


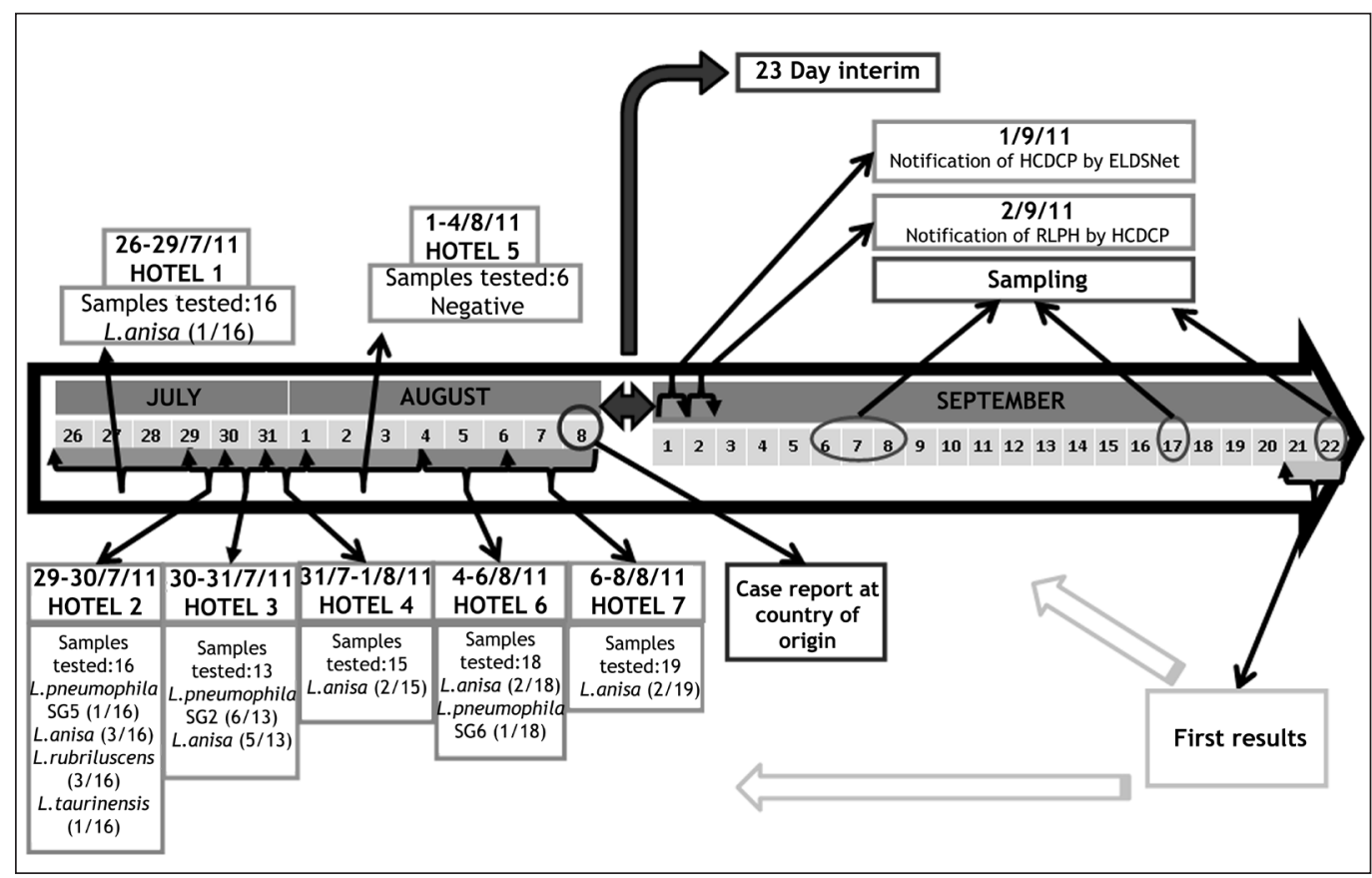

Fig. 1. Schematic representation of action timelines during case investigation.

Left part of the scheme: dates when tourist stayed at hotel and results of sampling procedure

Right part of the scheme: notification and sampling dates

and indirect cold and hot samples - after the water was flushed for one minute) were also collected from one randomly selected room, located very close to the water heater and one, located very far from the water heater. Where present, samples were also collected from pool showers and other decorative structures (waterfalls, spa facilities etc). The sampling procedure was carried out by the local public health authorities who followed the guidelines of EWGLINET (1).

A risk assessment (building age, chlorination procedure, pipe quality: iron or cooper made, water source: public water supply or borehole, water heater age, daily record keeping on hot water temperatures, chlorine concentration, etc. was also carried out at each hotel.

\section{Laboratory Investigation}

All water samples were stored at $4^{\circ} \mathrm{C}$ and sent to the Regional Laboratory of Public Health of Crete for processing, within 24 hours of collection. Isolation of Legionella from water samples was performed by culture according to ISO 11731 (1998) and ISO 11731-2 (2004).

\section{Identification}

Identification of suspected colonies was performed using two independent methods. Serotyping of the suspected colonies was performed using an agglutination test (SLIDEX ${ }^{\circledR}$ LegionellaKit, Biomérieux, Craponne, France), which allows the discrimination of L. pneumophila serogroup 1 from serogroups 2-14 and
L. anisa, while for the exact detection of each SG, serogroupspecific monovalent antisera were used (Pro-lab, Richmond Hill, Canada). All suspected colonies were also identified using MALDI-TOF mass spectrometry on a MALDI Biotyper (Microflex LT MALDI-TOF mass spectrometer) (Bruker Daltonics, Leipzig, Germany) equipped with a microSCOUT ion source. The identification of spectra was performed against the microbial database (v3.1.2.0) (Bruker Daltonics, Leipzig, Germany). Results were evaluated based on the manufacturer scoring scheme.

In addition to the above, Legionella isolates were further typed by PCR amplification using primers $8 \mathrm{UA}$ and $1485 \mathrm{~B}$ targeting a 1477-bp portion of the Legionella $16 \mathrm{~S}$ rRNA gene, and primers LegmipF/R, which produce a variable product of approximately $700 \mathrm{bp}$, as previously described (2).

Furthermore, samples positive for L. pneumophila were processed by sequence based typing (SBT) PCR using loci of the flaA, pilE, asd, mip, mompS, proA, and neuA genes according to the EWGLI scheme $(3,4)$. All data in the database of SBT were accessed from the EWGLI website*.

The positive PCR product of interest was purified (PCR product purification kit, Qiagen, Germany) and directly sequenced using the sequencer CEQ 8000 Beckman Coulter (Bioanalytica - Genotype, Athens). The sequencing primers used have been described either in the EWGLI database (SBT approach) or in past studies (2). The sequences revealed were processed using Chromas v1.49 and Lasergene Ver.7.1 software for viewing the chromatograms and editing of the retrieved nucleotide sequences. Checking for allelic profiles was done through the website of EWGLI*.

\footnotetext{
*http://www.ewgli.org/
} 
Table 1. Investigation of environmental samples from the hotels where the patient stayed for at least one night

\begin{tabular}{|c|c|c|c|c|c|c|c|}
\hline \multirow[b]{2}{*}{ Hotel } & \multicolumn{3}{|c|}{ Legionella species other than L. pneumophila } & \multicolumn{3}{|c|}{ L. pneumophila } & \multirow[b]{2}{*}{ Sources of $(+)$ samples } \\
\hline & Positive & $\%$ & Species & Positive & $\%$ & Serogroup & \\
\hline 1 & $1 / 16$ & 6.3 & L. anisa & $0 / 16$ & 0 & & Room shower \\
\hline 2 & $7 / 16$ & 43.8 & $\begin{array}{l}\text { L. rubrilucens, } \\
\text { L. taurinensis } \\
\text { L. anisa }\end{array}$ & $1 / 16$ & 6.3 & 5 & $\begin{array}{c}\text { Room shower } \\
\text { Swimming pool shower }\end{array}$ \\
\hline 3 & $5 / 13$ & 38.5 & L. anisa & $6 / 13$ & 46.2 & 2 & $\begin{array}{c}\text { Water tank } \\
\text { External shower }\end{array}$ \\
\hline 4 & $2 / 15$ & 13.3 & L. anisa & $0 / 15$ & 0 & & Swimming pool shower \\
\hline 5 & $0 / 6$ & 0 & & $0 / 6$ & 0 & & \\
\hline 6 & $2 / 18$ & 11.1 & L. anisa & $1 / 18$ & 5.6 & 6 & $\begin{array}{c}\text { Water leaving boiler } \\
\text { Water recycling of air cooling } \\
\text { system }\end{array}$ \\
\hline 7 & $2 / 19$ & 10.5 & L. anisa & $0 / 19$ & 0 & & Room shower \\
\hline Total & $19 / 103$ & 18.4 & & $8 / 103$ & 7.8 & & \\
\hline
\end{tabular}

\section{RESULTS}

\section{Water Samples}

Thirty per cent (31/103) of the collected water samples were found positive for Legionella spp. Out of 31 Legionella spp positive samples, $19(18.4 \%)$ were positive for non-pneumophila and $8(7.8 \%$ ) were positive for L. pneumophila (Table 1$)$. Of 7 hotels potentially implicated in the disease, $6(85.7 \%)$ were positive for Legionella spp, in at least one sample, while one hotel was negative in all samples tested.

In total, 31 isolates were identified as follows: L. pneumophila sg 2 (6/31), L. pneumophila sg 5 (1/31), L. pneumophila sg 6 (1/31), L. anisa (15/31), L. rubrilucens (3/31) and L. taurinensis (5/31). Legionella spp was isolated from room shower samples in three hotels. Swimming pool showers and/or external shower installments were also found positive for Legionella in three other hotels under investigation.

\section{Source Identification}

MALDI-TOF (score >2.2), PCR amplification and serotyping confirmed the presence of L. pneumophila sg 5 . In the meantime, results from the successful isolation and typing of L. pneumophila sg 5 from the patient's samples were rapidly communicated to the Greek authorities by the French Lab. The allelic profile of the isolate both from the patient and from an environmental sample was 5, 2, 22, 10, 6 and 25 for the corresponding genes fla $\mathrm{A}, p i l \mathrm{E}$, asd, mip, mompS and proA. The neuA could not be amplified. Based on the patient's sample typing results, the results from environmental investigation, the course of the patient through the seven hotels and the incubation period of the pathogen ( $\sim 2-10$ days), hotel 2 was identified as the source of infection.

\section{Risk Assessment Findings}

We did not manage to retrieve all the information required from all hotels from which samples were collected. Based on the data we collected, almost half (48.3\%) of the buildings aged more than 20 years, chlorination was performed at the majority (93\%) of the hotels, however, chlorine concentrations were $<0.2 \mathrm{ppm}$ in more than half of the samples $(63.7 \%)$, most pipes $(84.1 \%)$ were iron made, most hotel managers mentioned that they used public water supply as water source (although it was not very easy to discover hidden boreholes), the age of water heaters was $>10$ years in the majority of cases $(74.5 \%)$, and only in few hotels (especially those with $>$ three stars) was hot water temperature record kept on a daily basis.

\section{Control Measures}

Improvement notice was given to the seven hotels by the local authorities, soon after thermal and chemical disinfection of the plumbing systems was carried out by the hotels and was certified by the authorities. Re-sampling and re-testing of the hotels, one month following the investigation, was negative for Legionella. No other cases occurred at these hotels for the rest of the season. As regards hotel No 2 that was implicated to the human infection, re-sampling was performed for the following two years, again ending up in negative results.

\section{DISCUSSION}

Upon notification by ELSDnet of a single case of Legionnaire's disease in a French tourist, environmental investigations took place in seven hotels in order to identify the source of infection. Six hotels were found positive for Legionella, with 19/103 samples (18.4\%) positive for Legionella spp and 8 (7.8\%) positive for L. pneumophila (sgs 2, 5 and 6 identified). After communication with the French authorities, there was a successful typing match between the clinical and environmental samples leading to conclusion that hotel 2 was the source of infection. Of the seven genes used for SBT scheme, neuA was not made possible to get amplified; this is not strange and may be due to either heterogeneity of neuA sequence or its complete absence from some L. pneumophila non sg1 strains (5). 
During this travel associated Legionella case investigation, several worth mentioning issues came up. One of these issues is the time interval between the date the case was reported in the patients' country of origin (8 August) and the date the local Greek authorities began the source investigation ( 6 September). This time gap includes 25 days before the Greek authorities were notified of the travel case by ELDSNet and three additional days until the sample collection started. In total, 28 days passed from the day of report until the sampling day or 38 days from the reporting day until obtaining the first laboratory results (Fig. 1); a time interval that could have contributed to the occurrence of additional human cases. To ensure validity of the above observation we investigated all notifications concerning Legioniellosis cases in Crete sent to us from HCDCP. From 2011 to 2016, a total of 65 cases were reported from patients returning to their countries, implicating hotels in Crete. The average number of days elapsed from onset until notification of Greek authorities was 18 days (minimum 4, maximum 76). The days elapsed until the notification of the laboratory ranged from 0 to 20 (average of 4 days). Improvement in the speed of data exchange between ELDSNet partners should be considered in order to help to more efficient epidemiological investigations and to minimize new, but avoidable human cases.

Opposed to the notification delay of the case described herein, there was a very good and rapid communication between the Greek and French lab authorities. The need for communication between laboratories occurred while attempting to identify the source of infection. The data initially given through the ELDSNet network referred to the urine antigen test (UAT) result only. When the laboratory analysis was complete, no L. pneumophila sg1 was detected, thus we wondered if the positive UAT was a result due to intra-genus cross-reactivity as such occasions have been described previously $(6,7)$. Only after RLPHoC attempted communication with the Legionellosis Reference Centre of Lyon, we were informed that, besides UAT, there was a positive culture and that the strain was identified as L. pneumophila sg5. This condition raised further issues that deserve to be addressed such as the incorrect belief that a positive UAT involves only $L$. pneumophila $\operatorname{sg} 1$ and the need for more intensive attempts, and perhaps improvement of protocols to isolate the causative agent.

According to past reports, L. pneumophila $\mathrm{sg} 1$ is the most commonly identified pathogen (8). However, this does not recant the fact that other serogroups and species could also be implicated in the disease. Over the last decade, detection by UAT has become the most widely used test for confirming infection, but cultivation remains the gold standard for identifying the pathogen. The dissemination of UAT may have improved the number of cases diagnosed as L. pneumophila sg1, but without cultivation, other species and serogroups, which may account for up to $16 \%$ of Legionellosis cases, are missed out (9). Recent comparative studies showed that UAT from different producing companies achieve approximately $81 \%$ of sensitivity (10), with this percentage being the result from testing only patient samples diagnosed with L. pneumophila sg1. This well achieved sensitivity percentage can be dramatically decreased down to $37 \%$ when testing samples that contain L. pneumophila of variable serogroups (11).

Furthermore, the findings described herein add up to previous reports where L. pneumophila sgs other than sg1 have been detected using UATs $(6,7)$. Indeed, despite the high sensitivity and the fact that UAT has outplaced other laboratory methods for the diagnosis of Legionnaire's disease, intra-genus antigen cross-reactions do exist (12). These conditions raise the question about how clinicians and/or epidemiologists are certain that the case they are dealing with is a L. pneumophila sg1 when their only source of data is UAT. Even if a positive UAT is considered to be $100 \%$ sensitive and specific for L. pneumophila $\mathrm{sg} 1$, in the absence of the Sequence Based Typing (SBT) it is unsure whether a direct linkage can be made between the patient and the source of infection. In the latter years the option of SBT has been applied to most of L. pneumophila sgs as the analogous condition became apparent to the scientific world.

In order to perform serotyping isolation is needed. According to the European Working Group for Legionella Infections, since 1996 , only $10 \%$ of all cases in Europe have been culture-confirmed regardless of their travel history $(8,13)$. This has resulted in a very low proportion of matching isolates and linking cases with environmental findings. In our case, the detection of the source of infection would not have been possible in the absence of culture from clinical samples. However, to perform cultures, specimens of respiratory secretions including sputum should be routinely sent from patients with high severity community acquired pneumonia or where Legionnaires' disease is suspected on epidemiological or clinical grounds, regardless results of the urinary antigen test $(14,15)$. The facts described herein come in accordance with previous reports highlighting the importance of isolating and typing Legionella from as many clinical and environmental samples as possible to help identify the source of infection (16-18).

\section{CONCLUSION}

In conclusion, there is a need for ELDSNet to timely inform the network partners involved in a travel associated Legionella case investigation in order to minimize the occurrence of further cases and to allow for more accurate environmental results, closer to the time of infection. Furthermore, updating of the case-involved partners with information such as serogroup and/ or SBTs of the infecting strain, that were perhaps not available in the first case report, is essential. This is not the first case of a UAT to be positive with a $L$. pneumophila sg5 $(6,7)$ raising once again the question about the specificity of UATs and how sure someone can be about the source of infection based on absence of isolation and the UAT result only. This made even more apparent the need for more tests to be performed in a suspected case and raised the importance to perform the SBT scheme in both clinical and environmental samples in order to reinforce the evidence of the infection source. The above do not deny the importance of UATs for diagnostic use but renders them insufficient for detecting the source of patients' infection as well as possible infections by other serogroups and/or species.

\section{Acknowledgements}

We would like to thank Sophie Jarraud from the Centre National de Référence des légionelles, Site du CNR des légionelles and Christine Campèse from the Institut de Veille Sanitaire, Saint Maurice Cedex, France, for the assistance in providing information on the case, as well as the L. pneumophila isolation from the patient. 


\section{Conflict of Interest}

None declared

\section{REFERENCES}

1. Joseph C, Lee J, Surman-Lee S, Drasar V, Crespi S, Briand E. EWGLI technical guidelines for the investigation, control and prevention of travel associated Legionnaires' disease [Internet]. London: European Working Group for Legionella Infections; 2011 [cited 2016 Dec 20]. Available from: http://ecdc.europa.eu/en/healthtopics/legionnaires_disease/ELDSNet/Documents/EWGLI-Technical-Guidelines.pdf.

2. Ratcliff RM, Lanser JA, Manning PA, Heuzenroeder MW. Sequencebased classification scheme for the genus Legionella targeting the mip gene. J Clin Microbiol. 1998 Jun;36(6):1560-7.

3. Gaia V, Fry NK, Afshar B, Lück PC, Meugnier H, Etienne J, et al. Consensus sequence-based scheme for epidemiological typing of clinical and environmental isolates of Legionella pneumophila. J Clin Microbiol. 2005 May;43(5):2047-52.

4. Ratzow S, Gaia V, Helbig JH, Fry NK, Lück PC. Addition of neuA, the gene encoding $\mathrm{N}$-acylneuraminate cytidylyl transferase, increases the discriminatory ability of the consensus sequence-based scheme for typing Legionella pneumophila serogroup 1 strains. J Clin Microbiol. 2007 Jun;45(6):1965-8.

5. Farhat C, Mentasti M, Jacobs E, Fry NK, Lück C. The N-acylneuraminate cytidyltransferase gene, neuA, is heterogenous in Legionella pneumophila strains but can be used as a marker for epidemiological typing in the consensus sequence-based typing scheme. J Clin Microbiol. 2011 Dec;49(12):4052-8.

6. Kura F, Amemura-Maekawa J, Yagita K, Endo T, Ikeno M, Tsuji H, et al. Outbreak of Legionnaires' disease on a cruise ship linked to spa-bath filter stones contaminated with Legionella pneumophila serogroup 5 . Epidemiol Infect. 2006 Apr;134(2):385-91.

7. Lück PC, Schneider T, Wagner J, Walther I, Reif U, Weber S, et al. Community-acquired Legionnaires' disease caused by Legionella pneumophila serogroup 10 linked to the private home. J Med Microbiol. 2008 Feb;57(Pt 2):240-3
8. ECDC. Legionnaires' disease in Europe, 2010. Stockholm: European Centre for Disease Prevention and Control; 2012.

9. Chen DJ, Procop GW, Vogel S, Yen-Lieberman B, Richter SS. Utility of PCR, culture, and antigen detection methods for diagnosis of Legionellosis. J Clin Microbiol. 2015 Nov;53(11):3474-7.

10. Bruin JP, Diederen BM. Evaluation of Meridian TRU Legionella(R), a new rapid test for detection of Legionella pneumophila serogroup 1 antigen in urine samples. Eur J Clin Microbiol Infect Dis. 2013 Mar;32(3):333-4.

11. Svarrer CW, Lück C, Elverdal PL, Uldum SA. Immunochromatic kits Xpect Legionella and BinaxNOW Legionella for detection of Legionella pneumophila urinary antigen have low sensitivities for the diagnosis of Legionnaires' disease. J Med Microbiol. 2012 Feb;61(Pt 2):213-7.

12. Mercante JW, Winchell JM. Current and emerging Legionella diagnostics for laboratory and outbreak investigations. Clin Microbiol Rev. 2015 Jan;28(1):95-133.

13. Legionnaires' disease in Europe, 1996. Bull World Health Organ. 1998;76(2):213-7.

14. Naik FC, Ricketts KD, Harrison TG, Joseph CA. Legionnaires' disease in residents of England and Wales: 2007. Health Protection Rep. 2009;3:14.

15. Lim WS, Baudouin SV, George RC, Hill AT, Jamieson C, Le Jeune I, et al.; Pneumonia Guidelines Committee of the BTS Standards of Care Committee. BTS guidelines for the management of community acquired pneumonia in adults: update 2009. Thorax. 2009 Oct;64 Suppl 3:iiil-55.

16. Keramarou M, Evans MR; South Wales Legionnaires Disease Outbreak Control Team. A community outbreak of Legionnaires' disease in South Wales, August-September 2010. Euro Surveill. 2010 Oct 21;15(42). pii: 19691.

17. Kirrage D, Reynolds G, Smith GE, Olowokure B; Hereford Legionnaires Outbreak Control Team. Investigation of an outbreak of Legionnaires' disease: Hereford, UK 2003. Respir Med. 2007 Aug;101(8):1639-44.

18. Rota MC, Pontrelli G, Scaturro M, Bella A, Bellomo AR, Trinito MO, et al. Legionnaires' disease outbreak in Rome, Italy. Epidemiol Infect. 2005 Oct;133(5):853-9. 